\title{
A Sensor Based Assessment Monitoring System for Patients with Neurological Disabilities
}

\author{
Lu Bai* \\ School of Computing, Ulster Universitas, Belfast, UK \\ Email: 1.bai@ulster.ac.uk \\ *Corresponding Author
}

\begin{abstract}
The neurological conditions can cause the disability of upper limb and the rehabilitation therapy can help the patients to restore their upper limb motion. However, the current method for upper limb rehabilitation assessment is very basic. The aim of this work is to develop a system and visualize the information to support the doctors and clinicians in the assessment of upper limb motion of patients who are undertaken neurological rehabilitation. Movement tracking including position and orientation have been tracked and data analysis have been done in both time domain and frequency domain. Furthermore, movement smoothness analysis has been done to obtain more information from patients' movement recovery. The created information visualization can provide objective measurements of patients' motion recovery and insightful information and for doctors and clinicians including the frequency analysis and movement smoothness analysis. The findings showed the system is able to provide accurate position within $0.1 \mathrm{~cm}$ and orientation tracking within 1 degree and meaningful insights for the assessment of upper limb motion functions in daily rehabilitation assessment by providing doctors and clinicians with visualizations of the objective measurements.
\end{abstract}

Keywords-upper limb rehabilitation, inertial sensing, information visualization, wearable sensors

\section{NOMENCLATURE}

${ }^{0} P_{A} \quad$ Position of the joint $\mathrm{A}$ in the $X_{0}-Y_{0}$ reference frame

${ }^{0} P_{B} \quad$ Position of the joint $\mathrm{B}$ in the $X_{0}-Y_{0}$ reference frame

${ }^{0} P_{C} \quad$ Position of the joint $\mathrm{C}$ in the $X_{0}-Y_{0}$ reference frame

$\theta_{i} \quad$ The angle from $x_{i-1}$ to $x_{i}$ measure along $z_{i}$

\section{INTRODUCTION}

Neurological conditions and disorders are the most common cause of adult mental and physical disabilities. One outcome of acquiring neurological deficit can be dysfunction of upper limb function [1]. Rehabilitation, which usually includes occupational therapy and physiotherapy, can help to ease symptoms, and regain upper limb function [2,3]. Assessment of recovery is an important aspect of any rehabilitation program [4-6]. In general, most of the currently available assessments are viewed and scored by therapists based on assessment scales and decision rules. A number of clinical assessment rules of the motor function were widely used including Fugl-Meyer [7] and Motor Assessment Scale [8], Modified Ashworth Scale [9] and etc. However, these clinical scales do not provide objective data on the physical movement of the upper limb or lack of objective feedback on the recovery of function during the rehabilitation programs. The availability of a system to provide objective data on passive and active upper limb movement should help in the assessment of recovery and the evaluation of additional data provided by the system.

Choosing a suitable motion monitoring system for use in a clinic or general hospital poses restrictions on what is acceptable to the clinician as well as to the patient. Visual or video tracking systems are well proven for motion analysis and meet the requirements for upper limb tracking [10-12]. However, they are relatively complex, expensive, require careful setup and are not suited for use in a general hospital clinic. More recently, non-visual tracking technologies using inertial, mechanical, acoustic and magnetic sensing have been developed for more general clinical use. Of these technologies, inertial sensors and the application of kinematic modelling has demonstrated possible advantages for human motion tracking over other technologies in terms of effectiveness and operability [13-16]. Various studies have showed good accuracy in human upper limb motion tracking based on inertial measurement unit $[10,17]$. In addition, some gaming sensors have been used in the human motion analysis and rehabilitation assessment including Microsoft Kinect [18-21], and Nintendo Wii and Sony Move [22,23].

The aim of this study is to develop an upper limb motion monitoring system to provide doctors and clinicians with insightful information and visualizations about the patients' motor recovery in daily rehabilitation assessment during the rehabilitation programme. This assessment system should enable the clinicians to obtain repeatable and objective measurements of the progress and efficacy of the interventions in clinic. The proposed system and clinical tests were evaluated and validated in both lab settings and clinical settings in the previous studies [24-26]. A GUI tool has been developed to provide visualisations for the doctors and clinicians with a variety of objective assessments which are tested based on an inertial sensing system. It will also provide potential applications in home environment.

\section{METHODS}

In order to accurately track $3 \mathrm{D}$ position and orientation of the human upper limb motion, two position tracking algorithms are used including kinematic modelling and dead reckoning method (As seen in Fig. 1) based on the data obtained from accelerometer, gyroscope and magnetometer. Furthermore, frequency analysis and movement smoothness 
analysis have been done to provide additional insightful information. Details of these algorithms will be discussed in the subsections below.

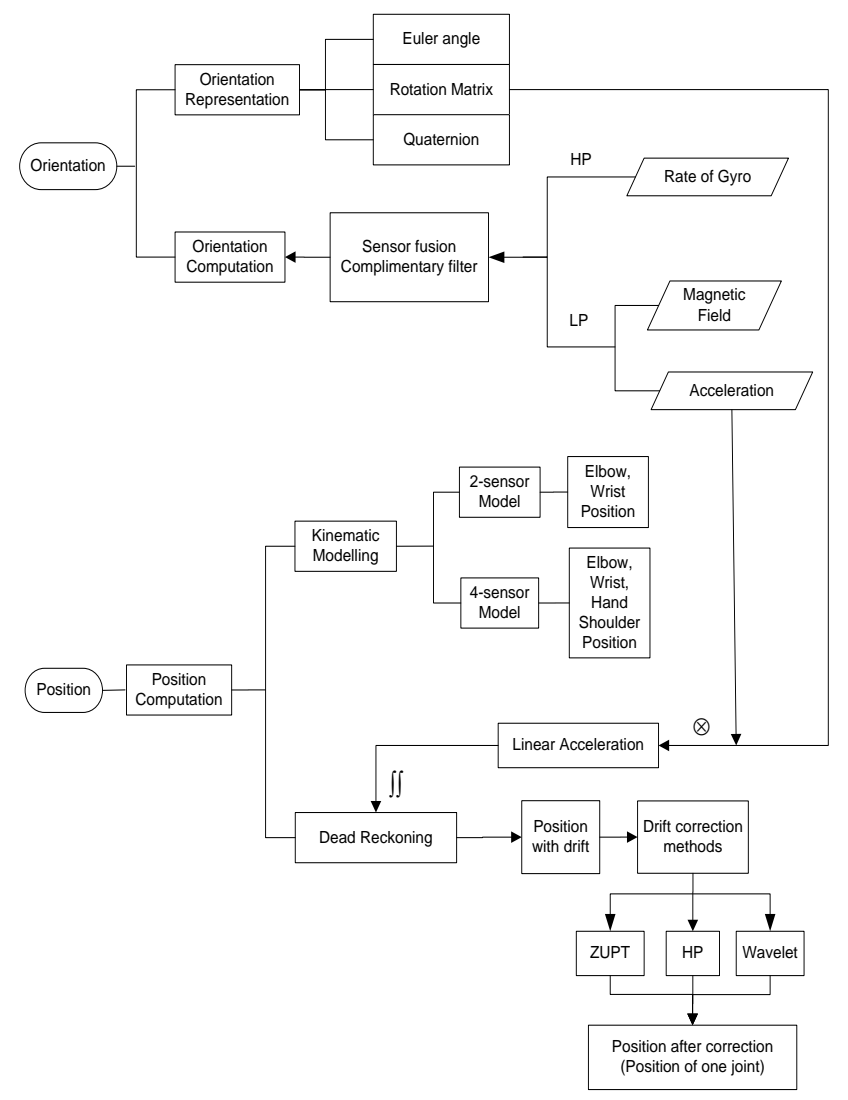

Fig. 1. Inertial tracking (Orientation and position tracking) algorithms used in this project

\section{A. Kinematic model}

The Kinematic model, which is based on the upper limb segments linked by joints, derives the position of each upper limb segment from the orientation outputs of the sensors on each limb segment [17]. Multiple sensors are required to build up the Kinematic model.

This two-link planar kinematic chain for the human arm is shown in Fig. 2. Points A, B and C in the figure represent three joints, the shoulder, elbow, and wrist, respectively. The reference frame transaction below uses the right-hand rule.

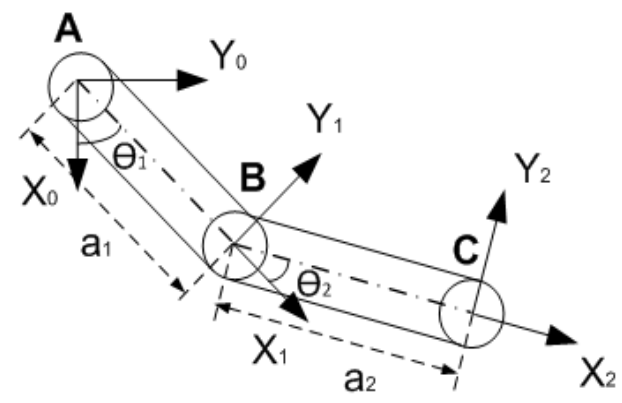

Fig. 2. A two-link planar model

The reference frame is established following the axis of the upper limb segments. The upper arm link transformation matrix has been established in the equations shown below by applying the D-H parameters [27] in the Equation (2) and Equation (3), ${ }^{1} P_{A},{ }^{1} P_{B}$ and ${ }^{2} P_{C}$ are the position data for the three joints, A, B and C. ${ }^{0} P_{A},{ }^{0} P_{B}$ and ${ }^{0} P_{C}$ represent the position data of joints $\mathrm{A}, \mathrm{B}$ and $\mathrm{C}$ in the reference frame $X_{0}-$ $Y_{0}$.

$$
\begin{aligned}
{ }^{0} P_{A} & =\left[\begin{array}{c}
x_{0} \\
y_{0} \\
z_{0} \\
1
\end{array}\right] \\
{ }^{0} P_{B} & =\left[\begin{array}{c}
{ }^{0} x_{1} \\
{ }^{0} y_{1} \\
{ }^{0} z_{1} \\
1
\end{array}\right]={ }_{1}^{0} T{ }^{1} P_{B}=\left[\begin{array}{cccc}
c_{1} & -s_{1} & 0 & a_{1} c_{1} \\
s_{1} & c_{1} & 0 & a_{1} s_{1} \\
0 & 0 & 1 & 0 \\
0 & 0 & 0 & 1
\end{array}\right] \times\left[\begin{array}{c}
{ }^{1} x_{1} \\
{ }^{1} y_{1} \\
1 \\
{ }^{1} z_{1} \\
1
\end{array}\right](2)
\end{aligned}
$$

Equation (2) represents the transformation of position of joint $\mathrm{B}\left({ }^{0} P_{B}\right)$ from the reference frame $X_{1}-Y_{1}$ to the position of joint B $\left({ }^{1} P_{B}\right)$ in the $X_{0}-Y_{0}$ reference frame.

$$
\begin{aligned}
& { }^{0} P_{C}=\left[\begin{array}{c}
{ }^{0} x_{2} \\
{ }^{0} y_{2} \\
{ }^{0} z_{2} \\
1
\end{array}\right]={ }_{2}^{0} T^{2} P_{C}={ }_{1}^{0} T_{2}^{1} T^{2} P_{C} \\
& =\left[\begin{array}{cccc}
c_{1} & -s_{1} & 0 & a_{1} c_{1} \\
s_{1} & c_{1} & 0 & a_{1} s_{1} \\
0 & 0 & 1 & 0 \\
0 & 0 & 0 & 1
\end{array}\right] \times\left[\begin{array}{cccc}
c_{2} & -s_{2} & 0 & a_{2} c_{2} \\
s_{2} & c_{2} & 0 & a_{2} s_{2} \\
0 & 0 & 1 & 0 \\
0 & 0 & 0 & 1
\end{array}\right] \times\left[\begin{array}{c}
{ }^{2} x_{2} \\
{ }^{2} y_{2} \\
{ }^{2} z_{2} \\
1
\end{array}\right]
\end{aligned}
$$

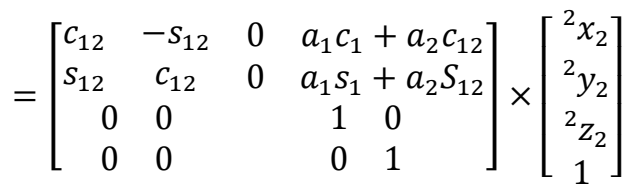

where $s_{1}=\sin \theta_{1}, s_{1}=\cos \theta_{1}, s_{12}=\sin \left(\theta_{1}+\theta_{2}\right)$ and $c_{12}=\cos \left(\theta_{1}+\theta_{2}\right)$. Equation (3) represents the transformation of position of joint $\mathrm{C}\left({ }^{0} P_{C}\right)$ from the reference frame $X_{2}-Y_{2}$ to the position of joint $\mathrm{C}\left({ }^{2} P_{C}\right)$ in the $X_{0}-Y_{0}$ reference frame.

To apply this model, the sensor orientation and the length of the arm segments are required inputs to the Kinematic model.

\section{B. Dead reckoning}

In order to explore the feasibility of using a single sensor to track the movement of one segment, the DR method [28] is used. The details of the implementation of this method can be found in the previous study [17]. The implementation of this method can potentially simplify the sensing system setup and reduce the cost of an upper limb motion monitoring system.

\section{Frequency domain analysis}

Pathological tremor can be correlated to specific diagnosis of some conditions such as Parkinson's disease and essential tremor, and is also useful in the assessment of the rehabilitation process. In the measurements made in this research, tremor usually most obviously presents itself in the acceleration data and also the accelerometer is sensitive in picking up the small movement. Analysis of the acceleration frequency spectrum should provide objective information on the amplitude and frequency content of the tremor. In the frequency analysis, the Fast Fourier Transform (FFT) has 
been used. Moreover, as the presence of low level tremors, which are difficult to detect using conventional screening, can precede the normal diagnosis of these conditions (e.g. Parkinson's disease and essential tremor) by several years [29], this analysis may prove to be a useful diagnostic tool.

\section{Movement smoothness analysis}

Movement smoothness is an important parameter used in assessing upper limb motion. And in evaluating the patient's motor recovery, which has been investigated in stroke patients [30-32]. Two useful parameters used in quantitative movement smoothness measurement are the Movement Unit Number (MUN) and the Normalised Jerk Score (NJS).

MUN is considered to be a useful parameter used in the quantitative measurement of upper limb motion [33,34] and can be used to describe the movement smoothness. It has been used to analyses the movement of different upper limb segments including upper arm, lower arm, shoulder and hand in this research. MUN is defined as the total number of zero crossings in the acceleration signal. A smaller value of MUN indicates a smoother movement (e.g. the visual presentation of a healthy volunteer) while a bigger value of MUN indicates a jerkier movement with a number of irregularities (e.g. a visual presentation of a patient).

The NJS has also been used to estimate the movement smoothness related to stroke patients' movement analysis [33]. It is an evaluation of the sudden change of the movement and it can also be used as an indication of the trajectory smoothness. A smaller value of the NJS indicates a smoother trajectory while a bigger value of NJS indicates a jerker movement trajectory. The evaluation of this variable had been carried out on both healthy volunteers and patients. Equation (4) [33] is used to calculate the NJS. In this equation the position data used to compute NJS is the estimation from the kinematic model. In the equation (4), $t 1$ and $t 2$ are start and end time for a period of movement respectively, $t$ is the movement time, 1 is movement distance, and $(\mathrm{x}, \mathrm{y}, \mathrm{z})$ is the position coordinates.

$$
N J S=\sqrt{0.5 * \int_{t_{1}}^{t_{2}}\left(\left(\frac{d^{3} x}{d t^{3}}\right)^{2}+\left(\frac{d^{3} y}{d t^{3}}\right)^{2}+\left(\frac{d^{3} z}{d t^{3}}\right)^{2}\right) * d t *\left(\frac{t^{5}}{l}\right)}
$$

\section{IMPLEMENTATION}

The system software was developed to provide the user with the option of using either of the two position tracking methods - the Kinematic modelling and the DR method. Data from multiple sensors is needed if the kinematic model is used while only data from one sensor is needed when using DR method. In the kinematic model, upper limb lengths (upper limb lengths are the inputs of this GUI) are used together with the Rotation matrix of the upper limb segments to compute the displacement of associated arm joints in the required reference frame - usually the trunk or shoulder reference frame. The DR method uses double integration method and applies drifts correction methods for position estimation. The data processing and analysis techniques have been described in the Method section.

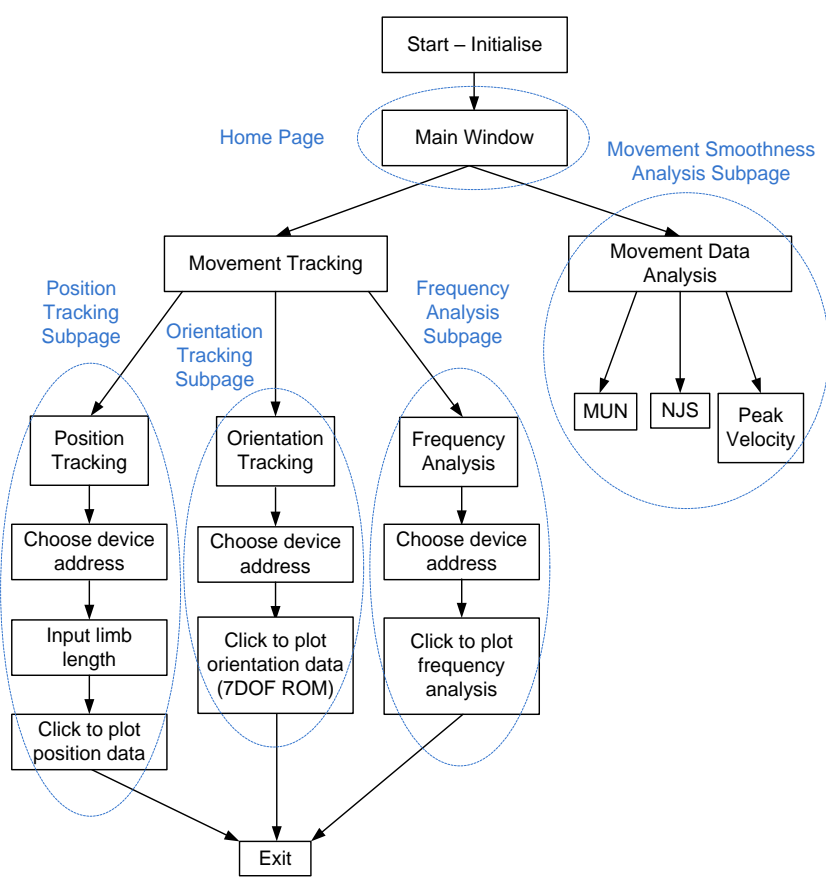

Fig. 3. Flowchart of the GUI interface

After the assessment has been recorded, the clinicians are able to continue to use this GUI to select and present the required data analysis. Fig. 3 shows the flowchart for this GUI. The main window contains the movement tracking and movement smoothness analysis. The position tracking, orientation tracking, and frequency analysis tracking subpages are included in the movement tracking. The above flow chart now has to be broken down into accessible GUI.

\section{A. Overview page}

From the home page, the user can implement basic movement data acquisition and analysis of upper limb movement data. In the overview page (See Fig. 4), the user faces a list of choices of different analysis including position tracking, orientation tracking, frequency analysis and movement smoothness analysis.

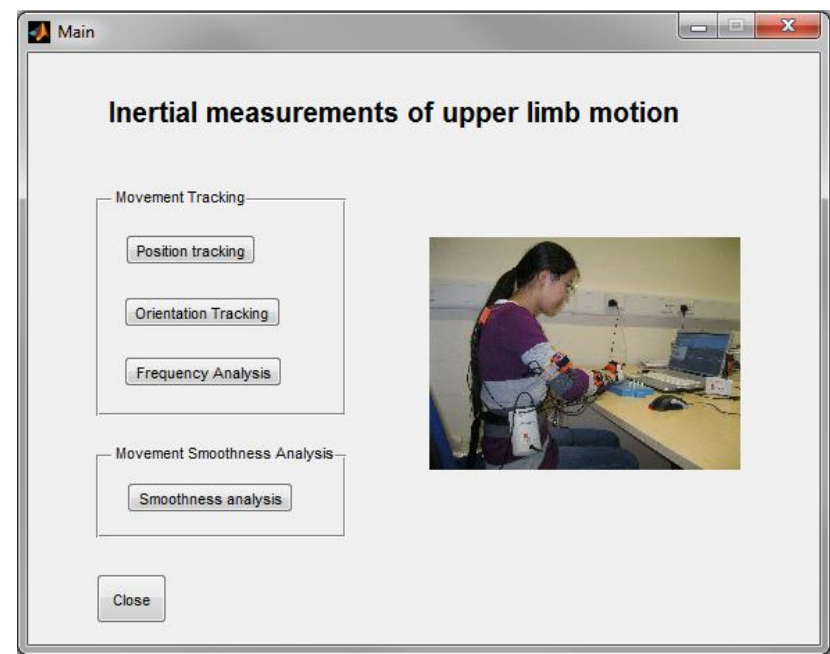

Fig. 4. The overview page 


\section{B. Position tracking - Kinematic model}

The Kinematic model and the DR method for position tracking are used here. In this position tracking GUI subpage, there are three options for upper limb position tracking as shown in Fig. 5. Two different tracking methods are available to users for different sensing systems including single sensor system and multiple sensors system.

Here data acquired using 4-sensors and data from a ninehole peg test is chosen as an example to explain the functions and operation. Initially, press the button MTx 1, MTx 2, MTx 3 , and MTx 4 to choose which sensor data that will be processed from the file directory as shown in Fig. 6.

And then input the upper arm and lower arm lengths in the edit text box since these two parameters are subjected dependent. The reference frame is in the trunk reference frame. Finally, the data can be displayed as 'Position Coordinate' and 'Movement Trajectory'. An example of the result plots is displayed in Fig. 7.

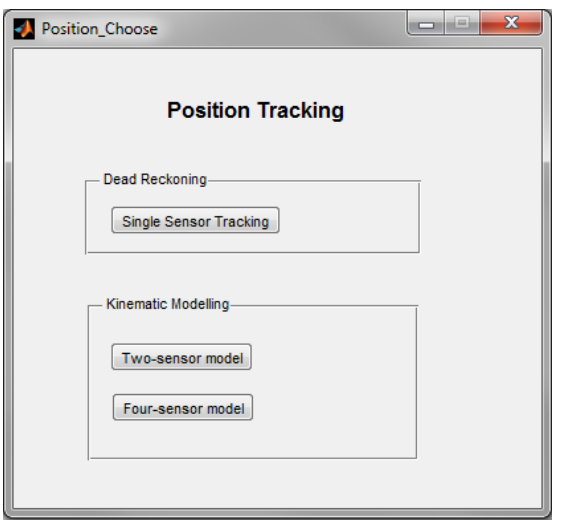

Fig. 5. Position tracking (Dead Reckoning \& Kinematic Modelling)

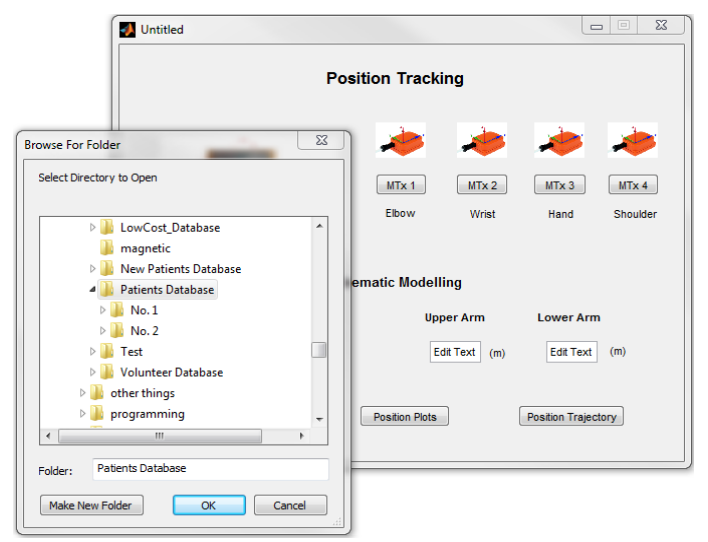

Fig. 6. Position tracking subpage (data selection from folder).

\section{Inertial measurements of Upper limb motion}
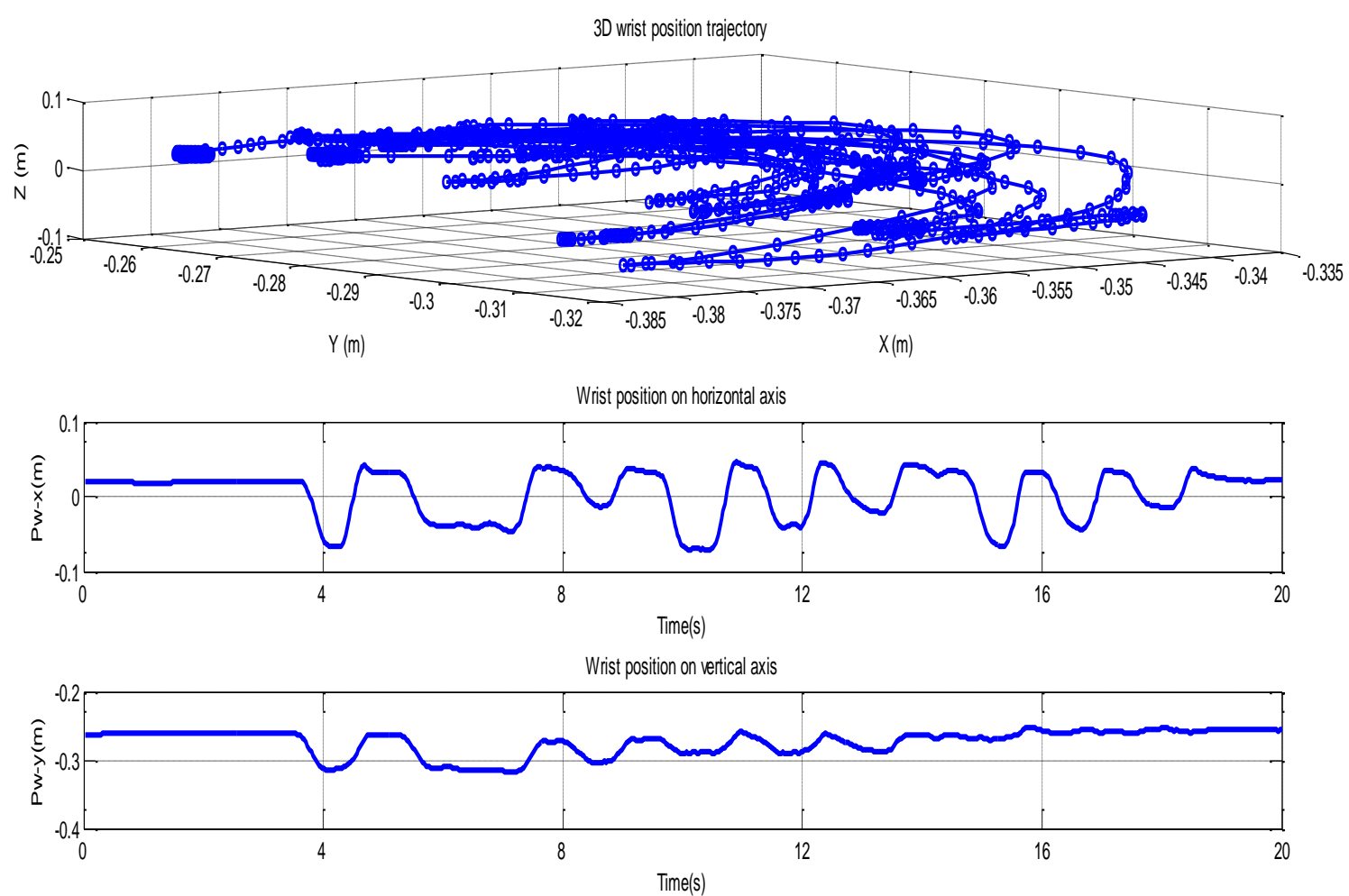

Fig. 7. Position coordinates and position trajectory plots for a nine-hole peg test 
The 3D trajectory tracking is displayed at the top (3D position trajectory on all three axes), the position tracking along the horizontal axis of the test table against time is in the middle and the position tracking along the vertical axis of the test table against time is at the bottom. The $3 \mathrm{D}$ trajectory indicates how the tracked segment moves through the 3D space. The position tracking on horizontal axis and vertical axis of the test table are of possible interest to clinicians since these position tracking results are related to the pegs location.

It should be noted that this is the first attempt at developing this analysis software. Fig. 7 only presents the basic data and may be difficult for the clinicians to interpret. Once the system has been evaluated and feedback obtained from the clinicians, the analysis software and how the data is presented can be further improved to meet the clinical requirements. Therefore, further analysis and evaluation is required - perhaps looking at related parameters such as movement smoothness.

\section{Position tracking - Dead reckoning}

After selecting the 'Single Sensor Tracking' button shown in Fig. 5, the single sensor position tracking is presented in Fig. 8. Similarly, as for the previous kinematic modelling subpage, the motion data of sensor is selected by clicking the button 'MTx1'. The computations of these three vectors use the DR method to minimize the drift in estimating the linear velocity and position.

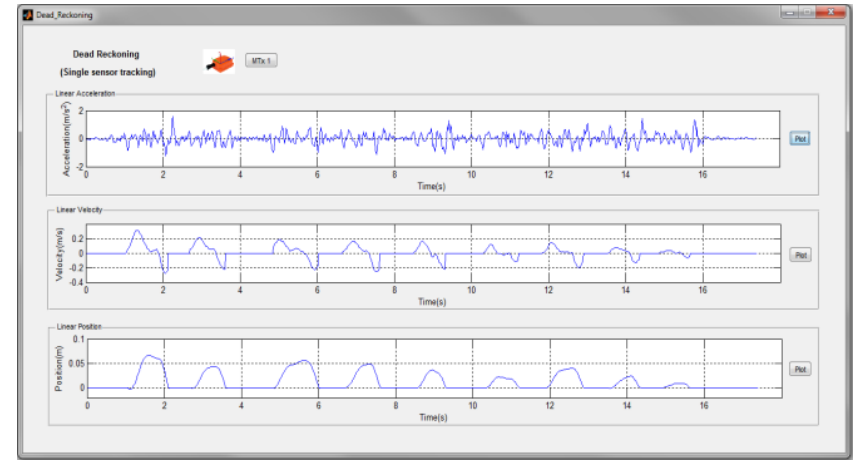

Fig. 8. Single Sensor Position tracking (uses Dead Reckoning)

Again the presentation of these computations is probably too detailed for easy analysis by the clinician. At present automated measurement of some of the timing and magnitude parameters has been implemented. However as is the case for the analysis of the data gained from the kinematic model, further evaluation will be required to identify what analysis the clinician thinks are important and how that data should be presented.

\section{Movement Tracking - Orientation Tracking}

Limb segment orientation and changes in that orientation with time is one of the important parameters identified by the clinician. In total, three sensors are currently used to track the orientation of the upper and forearm segments of the upper limb. The orientation analysis subpage is shown in Fig. 9.

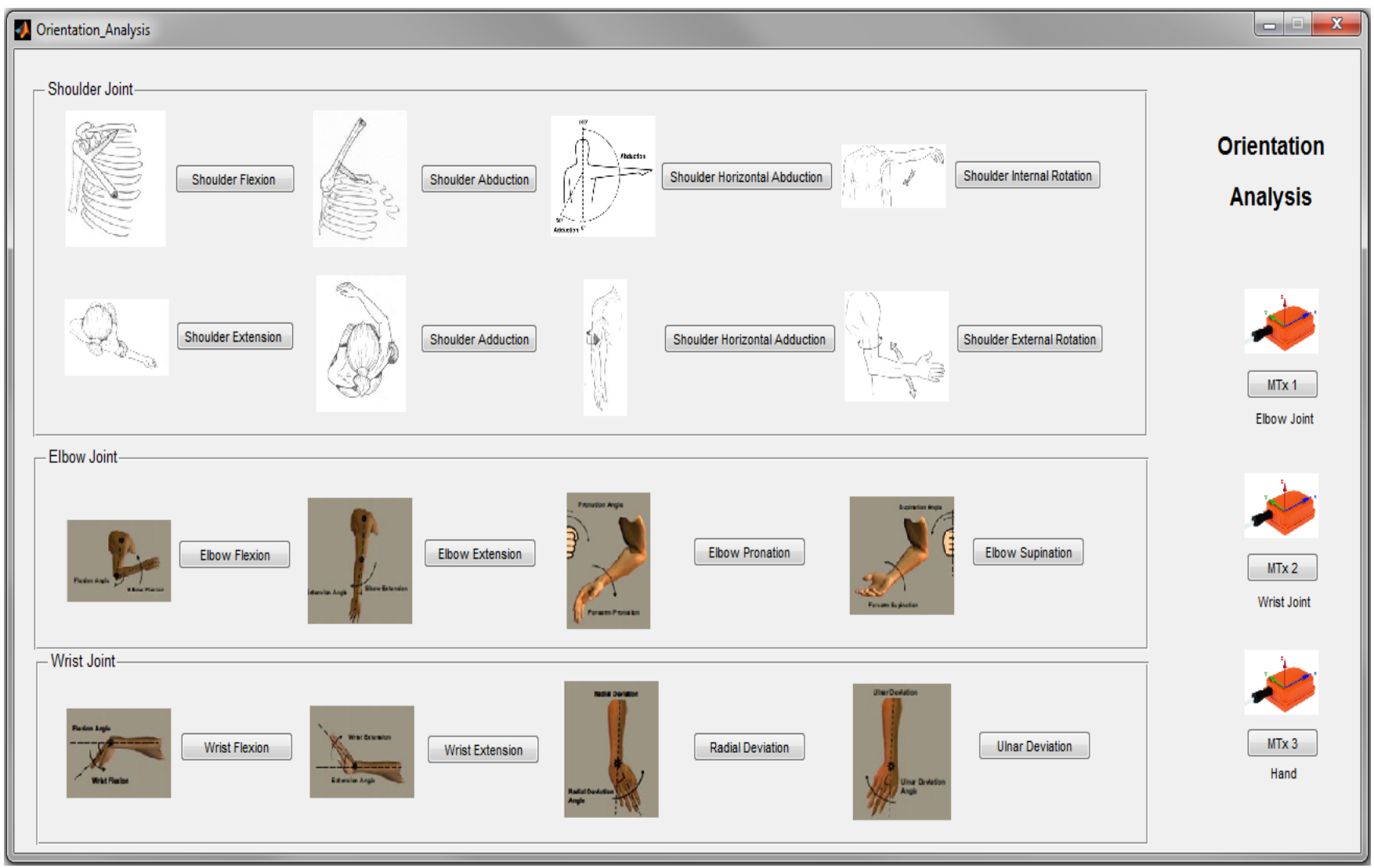

Fig. 9. Orientation tracking subpage 
Any of the orientations related to the 7 DOF range of motion tracked by this measurement system can be selected here. The orientation tracking for each segment can be analysed and displayed by clicking the corresponding 'joint' buttons. The directories of patient measurement files are then presented and the required data file can be selected. Fig. 10 shows an example of a single measurement of the shoulder abduction range of motion from a healthy volunteer. It can be seen that the movement is very smooth and the range of motion of the abduction is around 160 degrees.

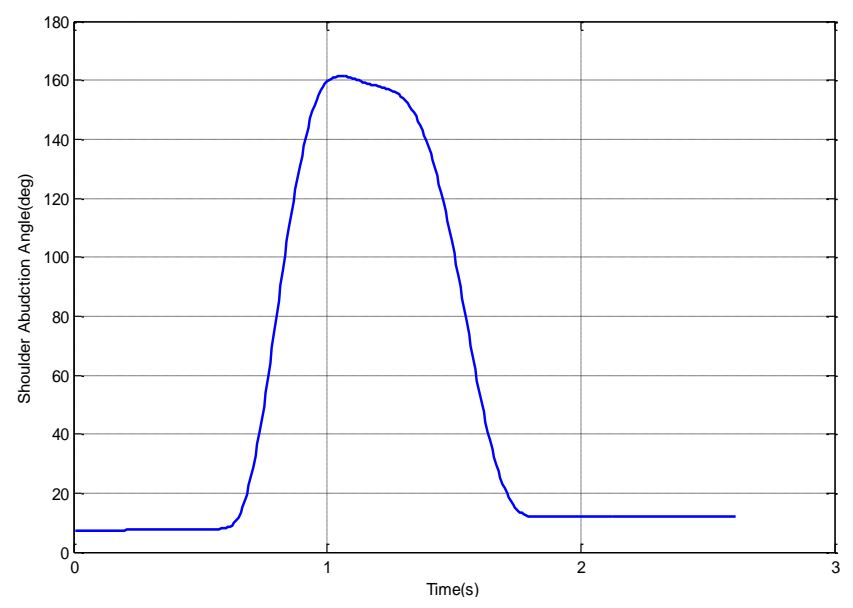

Fig. 10. Orientation tracking plot for healthy volunteer's shoulder abduction range of motion

\section{E. Movement Tracking - Frequency Analysis}

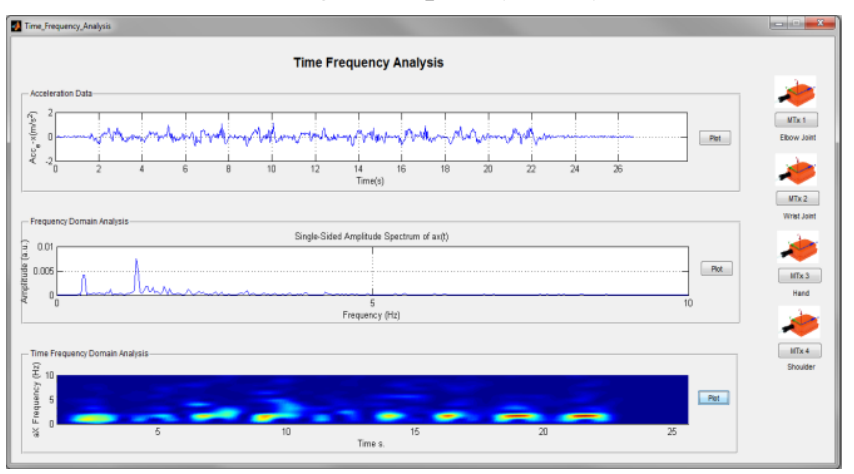

Fig. 11. Frequency analysis subpage (frequency analysis of a nine-hole peg test)

A basic frequency analysis option can be selected from the home page (Fig. 4). The frequency analysis is carried out on the linear acceleration data. Before the analysis, the user should choose the sensor which will be analyzed and click the corresponding 'MTx' button at the right side of this subpage to choose the data file. Then click the 'plot' button, the acceleration, its frequency spectrum and spectrogram will be plotted in the picture boxes respectively. This is a very basic analysis as it is not possible to select the time period of which the analysis is made. If this analysis proves to be of use, then this feature can be further developed. An example of this analysis is shown in Fig.11. The results shown in Fig. 11 is from a nine-hole peg test. Both the frequency spectrum and spectrogram are presented. From the spectrogram, the start and the end of the nine peg movements can be clearly seen and the frequency amplitude is thought to be correlated to the energy of the motion.
The linear acceleration is used here for the frequency analysis and the resultant frequency spectrum and spectrogram plots presented. The spectrogram is able to provide both frequency information and time information in one plot, and therefore, it can give extra information to the clinicians on the change of the patient's upper limb movement frequency against time. If this does prove to be the case, then the way in which the data is presented will require some refining in order to speed up analysis by the clinician.

\section{F. Movement Smoothness Data analysis}

In the method section, the idea that the measurement of movement smoothness by calculating MUN and NJS could help evaluate the patient's motor recovery was discussed. This GUI, as shown in Fig. 12, enables the user to obtain the calculated MUN and NJS for all the upper limb joints including elbow, wrist, hand and shoulder.

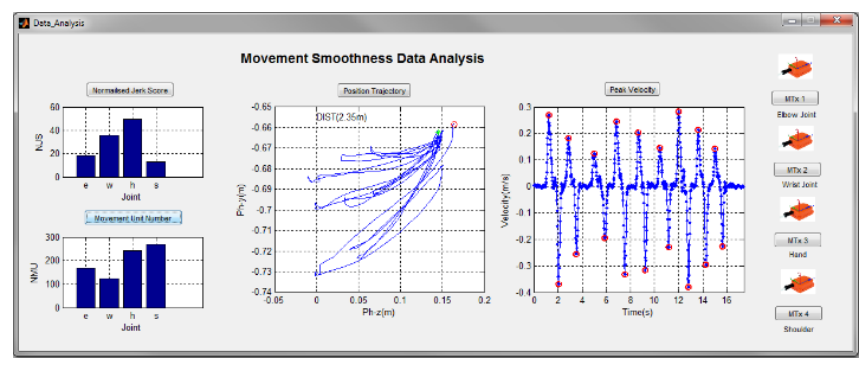

Fig. 12. Movement Smoothness Data analysis subpage

The user needs to re-select the MTx sensor which is to be analyzed after the previous analysis. This data can be plotted by selecting the 'Normalized Jerk Score' and 'Movement Unit Number' buttons on the top of this subpage. The values of NJS and MUN have been computed for different four segments. The position trajectory with start (red dot) and end (green dot) points are marked in the plot. In Fig. 12, the position trajectory is the result from a nine-hole peg test. In order to help the clinician, relate these scores to the actual movement the position trajectory is plotted and velocity data are also displayed on the same page. In this case the peak velocity is plotted in Fig. 12, in which the positive and negative peak velocity of the plot is marked with red dots.

If necessary other parameters such as position against time could be plotted if that helps the clinician understand the relationship between the jerk and movement scores with the recorded movement trajectory. In addition, the value of the position trajectory would be useful and can be a good estimation of the movement smoothness when compared with the ideal value of the position trajectory.

\section{CONCLUSION}

This paper presents the GUI development to enable the technical user to access and display some of the basic analysis parameters. Three subsections have been developed to enable the basic display of the position, orientation and frequency tracking data. By using this GUI, the user can carry out a basic analysis of the stored patients' data. The plotting of the position and range of motion (orientation) graph will give the clinicians an alternative view of the patients' movement. The frequency analysis can be a very useful tool in analysing the tremor patients. The movement smoothness data (data analysis subpage) analysis may give an easy quantitative 
analysis of the patients' movement performance recovery. Unlike the traditional assessment (rating scales) that simplify the measurement result to a value to describe patient's performance, this visualisation design gives the clinicians a different presentation of movement data in which the movement plot (e.g. position tracking, and range of motion tracking against time) gives a visualised way in presenting the measurements. It helps the clinicians in understanding and remembering the movement details of the patients during the assessment e.g. a missed peg in nine-hole peg test. The parameters such as MUN and NJS can be used to describe the movement smoothness which can be used as the objective assessment of the upper limb motion.

The comparable clinical assessment scales such as Disability Assessment Score and motor Assessment Scale can be included in the future development. Also in future design, more useful quantitative values can be presented in order to help evaluate the patients' upper limb performance through the GUI along with the kinematic measurement plots.

\section{REFERENCES}

[1] The Neurological Alliance, Neuro numbers, 2004.

[2] P. Langhorne, J. Bernhardt, G. Kwakkel, Stroke rehabilitation, Lancet. (2011). https://doi.org/10.1016/S0140-6736(11)60325-5.

[3] A. Pollock, S.E. Farmer, M.C. Brady, P. Langhorne, G.E. Mead, J. Mehrholz, F. van Wijck, Interventions for improving upper limb function after stroke, Cochrane Database Syst. Rev. (2014). https://doi.org/10.1002/14651858.CD010820.pub2.

[4] A. Houwink, R.H. Nijland, A.C. Geurts, G. Kwakkel, Functional recovery of the paretic upper limb after stroke: Who regains hand capacity?, Arch. Phys. Med. Rehabil. (2013). https://doi.org/10.1016/j.apmr.2012.11.031.

[5] Z. Zhang, Q. Fang, X. Gu, Objective assessment of upper-limb mobility for poststroke rehabilitation, IEEE Trans. Biomed. Eng. (2016). https://doi.org/10.1109/TBME.2015.2477095.

[6] N. Nordin, S.Q. Xie, B. Wünsche, Assessment of movement quality in robot- Assisted upper limb rehabilitation after stroke: A review, J. Neuroeng. Rehabil. (2014). https://doi.org/10.1186/1743-0003-11137.

[7] A.R. Fugl Meyer, L. Jaasko, I. Leyman, The post stroke hemiplegic patient. I. A method for evaluation of physical performance, Scand. J. Rehabil. Med. (1975).

[8] J.H. Carr, R.B. Shepherd, L. Nordholm, D. Lynne, Investigation of a new motor assessment scale for stroke patients, Phys. Ther. (1985). https://doi.org/10.1093/ptj/65.2.175.

[9] R.W. Bohannon, M.B. Smith, Interrater reliability of a modified Ashworth scale of muscle spasticity, Phys. Ther. (1987). https://doi.org/10.1093/ptj/67.2.206.

[10] A. Filippeschi, N. Schmitz, M. Miezal, G. Bleser, E. Ruffaldi, D. Stricker, Survey of motion tracking methods based on inertial sensors: A focus on upper limb human motion, Sensors (Switzerland). (2017). https://doi.org/10.3390/s17061257.

[11] C. Yang, A. Kerr, V. Stankovic, L. Stankovic, P. Rowe, S. Cheng, Human Upper Limb Motion Analysis for Post-Stroke Impairment Assessment Using Video Analytics, IEEE Access. (2016). https://doi.org/10.1109/ACCESS.2016.2523803.

[12] A.O. Bălan, L. Sigal, M.J. Black, A quantitative evaluation of videobased 3D person tracking, in: Proc. - 2nd Jt. IEEE Int. Work. Vis. Surveill. Perform. Eval. Track. Surveillance, VS-PETS, 2005. https://doi.org/10.1109/VSPETS.2005.1570935.

[13] H. Zhou, T. Stone, H. Hu, N. Harris, Use of multiple wearable inertial sensors in upper limb motion tracking, Med. Eng. Phys. (2008). https://doi.org/10.1016/j.medengphy.2006.11.010.

[14] B. Hingtgen, J.R. McGuire, M. Wang, G.F. Harris, An upper extremity kinematic model for evaluation of hemiparetic stroke, J. Biomech. (2006). https://doi.org/10.1016/j.jbiomech.2005.01.008

[15] J.C. Perry, J. Rosen, S. Burns, Upper-limb powered exoskeleton design, in: IEEE/ASME Trans. Mechatronics, 2007.
https://doi.org/10.1109/TMECH.2007.901934.

[16] L. Bai, M.G. Pepper, Y. Yan, S.K. Spurgeon, M. Sakel, M. Phillips, A multi-parameter assessment tool for upper limb motion in neurorehabilitation, in: Conf. Rec. - IEEE Instrum. Meas. Technol. Conf., 2011. https://doi.org/10.1109/IMTC.2011.5944169.

[17] L. Bai, M.G. Pepper, Y. Yan, S.K. Spurgeon, M. Sakel, M. Phillips, Quantitative Assessment of Upper Limb Motion in Neurorehabilitation Utilizing Inertial Sensors, IEEE Trans. Neural Syst. Rehabil. Eng. https://doi.org/10.1109/TNSRE.2014.2369740.

[18] G.M. Moreira, L.H.F. Giovanini, M.P.R. de Castro, G.N. Nogueira, T.C. Boumer, E.F. Manffra, Filtering motion signals from Microsoft Kinect ${ }^{\circledR}$ in the context of stroke rehabilitation, Res. Biomed. Eng. (2019). https://doi.org/10.1007/s42600-019-00029-8.

[19] C. Zerpa, C. Lees, P. Patel, E. Pryzsucha, The Use of Microsoft Kinect for Human Movement Analysis, Int. J. Sport. Sci. (2015).

[20] Y. Tian, X. Meng, D. Tao, D. Liu, C. Feng, Upper limb motion tracking with the integration of IMU and Kinect, Neurocomputing. (2015). https://doi.org/10.1016/j.neucom.2015.01.071

[21] S. Giancola, A. Corti, F. Molteni, R. Sala, Motion capture: An evaluation of kinect V2 body tracking for upper limb motion analysis, in: Lect. Notes Inst. Comput. Sci. Soc. Telecommun. Eng. LNICST, 2017. https://doi.org/10.1007/978-3-319-58877-3_39.

[22] L. Bai, M.G. Pepper, Y. Yana, S.K. Spurgeon, M. Sakel, Application of low cost inertial sensors to human motion analysis, in: 2012 IEEE I2MTC - Int. Instrum. Meas. Technol. Conf. Proc., 2012. https://doi.org/10.1109/I2MTC.2012.6229349.

[23] L. Bai, M.G. Pepper, Y. Yan, M. Phillips, M. Sakel, Low Cost Inertial Sensors for the Motion Tracking and Orientation Estimation of Human Upper Limbs in Neurological Rehabilitation, IEEE Access. (2020). https://doi.org/10.1109/ACCESS.2020.2981014.

[24] Lu Bai, Matthew G Pepper, Yong Yan, Malcolm Phillips, Mohamed Sakel, Inertial sensor based quantitative assessment of upper limb range of motion and functionality before and after botulinum toxin: a pilot study, Glob. J. Eng. Technol. Adv. (2020). https://doi.org/10.30574/gjeta.2020.2.3.0008.

[25] L. Bai, M.G. Pepper, Y. Yan, M. Phillips, M. Sakel, Quantitative measurement of upper limb motion pre- and post-treatment with Botulinum Toxin, Meas. J. Int. Meas. Confed. (2021). https://doi.org/10.1016/j.measurement.2020.108304.

[26] L. Bai, M. Pepper, Y. Yan, S.K. Spurgeon, M. Sakel, M. Phillips, Quantitative Assessment of Limb Motion by Inertial Sensors Before and After Botulinum Toxin for Spasticity, Arch. Phys. Med. Rehabil. (2014). https://doi.org/10.1016/j.apmr.2014.07.205.

[27] J.J. Craig, Manipulator-Mechanism Design, in: Introd. to Robot. Mech. Control, 2005.

[28] A. Lawrence, Modern Inertial Technology: Navigation, Guidance, and Control, 2nd ed, Mod. Inert. Technol. (1998).

[29] J. Jankovic, J. Beach, K. Schwartz, C. Contant, Tremor and longevity in relatives of patients with parkinson's disease, essential tremor, and control subjects, Neurology. (1995). https://doi.org/10.1212/WNL.45.4.645.

[30] B. Rohrer, S. Fasoli, H.I. Krebs, R. Hughes, B. Volpe, W.R. Frontera, J. Stein, N. Hogan, Movement smoothness changes during stroke recovery, J. Neurosci. (2002). https://doi.org/10.1523/jneurosci.2218-08297.2002.

[31] L. Dipietro, H.I. Krebs, S.E. Fasoli, B.T. Volpe, N. Hogan, Submovement changes characterize generalization of motor recovery $\begin{array}{lll}\text { after } & \text { stroke, } & \text { Cortex. }\end{array}$ https://doi.org/10.1016/j.cortex.2008.02.008.

[32] N. Hogan, D. Sternad, Sensitivity of smoothness measures to movement duration, amplitude, and arrests, J. Mot. Behav. (2009). https://doi.org/10.3200/35-09-004-RC.

[33] C.C. Tsao, M.M. Mirbagheri, Upper limb impairments associated with spasticity in neurological disorders, J. Neuroeng. Rehabil. (2007). https://doi.org/10.1186/1743-0003-4-45.

[34] E.Z. Tronick, L. Fetters, K.L. Olson, Y. Chen, Similar and Functionally Typical Kinematic Reaching Parameters in 7- and 15Month-Old in Utero Cocaine-Exposed and Unexposed Infants, Dev. Psychobiol. (2004). https://doi.org/10.1002/dev.20002. 